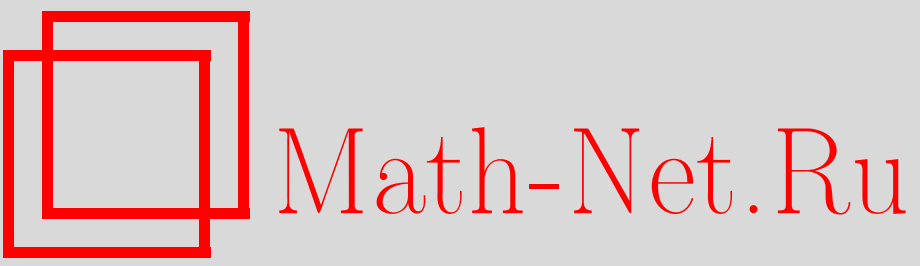

А. В. Щепетилов, Задача двух тел на пространствах постоянной кривизны. I. Связь гамильтониана с группой симметрий и редукция классической системы, TMФ, 2000, том 124, номер 2, 249-264

DOI: https://doi.org/10.4213/tmf637

Использование Общероссийского математического портала Math-Net.Ru подразумевает, что вы прочитали и согласны с пользовательским соглашением

http://www.mathnet.ru/rus/agreement

Параметры загрузки:

IP: 54.162 .85 .209

26 апреля 2023 г., 03:47:16 
ТЕОРЕТИЧЕСКАЯ

И МАТЕМАТИЧЕСКАЯ

ФИЗИКА

Том 124, № 2

август, 2000

(C) 2000 г.

\section{ЗАДАЧА ДВУХ ТЕЛ НА ПРОСТРАНСТВАХ ПОСТОЯННОЙ КРИВИЗНЫ. І. СВЯЗЬ ГАМИЛЬТОНИАНА С ГРУППОЙ СИММЕТРИЙ И РЕДУКЦИЯ КЛАССИЧЕСКОЙ СИСТЕМЫ}

Рассмотрена задача двух тел с центральным взаимодействием на односвязных пространствах постоянной кривизны произвольной размерности. Получено явное выражение квантового гамильтониана через радиальный дифференциальный оператор и генераторы групп изометрий конфигурационного пространства. Дано описание приведенной классической механической системы на однородном пространстве группы Ли в терминах орбит коприсоединенного представления этой группы. Получено описание приведенной классической задачи двух тел.

\section{1. ВВЕДЕНИЕ}

Односвязные пространства постоянной кривизны $\mathbb{S}^{n}$ и $\mathbb{H}^{n}$ обладают столь же широкими группами изометрий, как евклидово пространство $\mathbb{E}^{n}$, и не имеют выделенных точек и направлений [1]. Геодезический поток на этих пространствах эквивалентен движению классической частицы в евклидовом пространстве в кулоновском поле на поверхности постоянной энергии [2-5]. Обзор исследований одночастичной задачи в случаях классической и квантовой механики в поле центрального потенциала на таких пространствах содержится в работе [6]. Отметим здесь только работы [7-10], не указанные в [6].

Фазовые пространства двухчастичных задач $\mathbb{S}^{n} \times \mathbb{S}^{n}$ и $\mathbb{H}^{n} \times \mathbb{H}^{n}$, в отличие от евклидова случая, не являются пространствами постоянной кривизны. Априори в группу симметрий этих задач входят только изометрии пространства, сохраняющие потенциал взаимодействия. Эта группа слишком бедна, для того чтобы обеспечить интегрируемость двухчастичных задач. В то же время для нетривиальных потенциалов неизвестны какие-либо "скрытые" симметрии или другие механизмы интегрируемости. Более того, численные эксперименты, проведенные в работах $[11,12]$, свидетельствуют о неинтегрируемости классической ограниченной задачи двух тел на двумерной сфере с наиболее естественными потенциалами.

\footnotetext{
* Московский государственный университет, Москва, Россия. E-mail: alexey@quant.phys.msu.su
} 
Классическая механическая двухчастичная задача впервые была рассмотрена в работе [6], где для исключения движения системы в целом был использован метод гамильтоновой редукции систем с симметриями [13]. С помошью явных аналитических координатных вычислений на ЭВМ было получено описание редуцированных механических систем, проведена их классификация и получены условия сушествования глобальной динамики. В работе [14] впервые была рассмотрена аналогичная квантово-механическая задача в двумерном случае, т.е. на пространствах $\mathbb{S}^{2}$ и $\mathbb{H}^{2}$. Квантово-механический гамильтониан был выражен через генераторы групп изометрий и радиальный дифференциальный оператор. При этом оказалось, что структура последнего выражения напоминает структуру приведенной функции Гамильтона. Этот факт наводит на мысль о сушествовании обшей процедуры использования имеющейся групшы симметрий для одновременного упрощения классической и квантовой задач без использования громоздких вычислений. Такая процедура найдена в настояшей работе. Полученное с ее помощью выражение для квантово-механическогогамильтониана может быть использовано, по крайней мере, в трех задачах.

Во-первых, из этого выражения может быть получена функция Гамильтона приведенной классической механической системы. При этом используется описание редуцированной классической механической системы на однородном пространстве группы Ли в терминах орбит коприсоединенного представления этой группы (см. раздел 4). Во-вторых, данное выражение гамильтониана позволяет доказать самосопряженность двухчастичных гамильтонианов с сингулярным взаимодействием. В-третьих, используя теорию представления групп, можно свести задачу нахождения энергетических уровней гамильтониана к последовательности систем обыкновенных дифференциальных уравнений, нумеруемых неприводимыми представлениями группы изометрий. Двум последним задачам будет посвящена следующая работа.

\section{2. ОБОЗНАЧЕНИЯ}

Реализуем сферу $\mathbb{S}^{n}$ как пространство $\mathbb{R}^{n} \cup\{\infty\}$ с метрикой

$$
g_{s}=\left(4 R^{2} \sum_{i=1}^{n} d x_{i}^{2}\right) /\left(1+\sum_{i=1}^{n} x_{i}^{2}\right)^{2}
$$

где $x_{i}, i=1, \ldots, n,-$ декартовы координаты в $\mathbb{R}^{n}, R$ - радиус кривизны. Пусть $\rho^{s}(\cdot, \cdot)-$ расстояние между двумя точками $\mathbb{S}^{n}$. Связная компонента группы изометрий пространства $\mathbb{S}^{n}$, действуюшая слева, есть $\mathrm{SO}(n+1)$, а поля Киллинга на $\mathbb{S}^{n}$ имеют вид

$$
\begin{aligned}
X_{i j}^{s} & =x_{i} \frac{\partial}{\partial x_{j}}-x_{j} \frac{\partial}{\partial x_{i}}, \quad 1 \leqslant i<j \leqslant n, \\
Y_{i}^{s} & =\frac{1}{2}\left(1+x_{i}^{2}-\sum_{\substack{j=1 \\
j \neq i}}^{n} x_{j}^{2}\right) \frac{\partial}{\partial x_{i}}+x_{i} \sum_{\substack{j=1 \\
j \neq i}}^{n} x_{j} \frac{\partial}{\partial x_{j}}, \quad i=1, \ldots, n,
\end{aligned}
$$

и соответствуют некоторому базису в алгебре so $(n+1)$. 
Гиперболическое пространство $\mathbb{H}^{n}$ реализуем в единичном шаре $D^{n} \subset \mathbb{R}^{n}$, наделив его метрикой

$$
g_{h}=\left(4 R^{2} \sum_{i=1}^{n} d x_{i}^{2}\right) /\left(1-\sum_{i=1}^{n} x_{i}^{2}\right)^{2}, \quad \sum_{i=1}^{n} x_{i}^{2}<1 .
$$

Расстояние между двумя точками пространства $\mathbb{H}^{n}$ обозначим $\rho^{h}(\cdot, \cdot)$. Связная компонента группы изометрий, действуюшая слева, в этом случае есть группа $\mathrm{SO}(1, n)$ с алгеброй Ли so $(1, n)$, а поля Киллинга имеют вид

$$
\begin{aligned}
X_{i j}^{h} & =x_{i} \frac{\partial}{\partial x_{j}}-x_{j} \frac{\partial}{\partial x_{i}}, \quad 1 \leqslant i<j \leqslant n \\
Y_{i}^{h} & =\frac{1}{2}\left(1-x_{i}^{2}+\sum_{\substack{j=1 \\
j \neq i}}^{n} x_{j}^{2}\right) \frac{\partial}{\partial x_{i}}-x_{i} \sum_{\substack{j=1 \\
j \neq i}}^{n} x_{j} \frac{\partial}{\partial x_{j}}, \quad i=1, \ldots, n .
\end{aligned}
$$

\section{3. ПРЕДСТАВЛЕНИЯ СВОБОДНЫХ ГАМИЛЬТОНИАНОВ}

Рассмотрим конфигурационные пространства задачи двух тел $Q_{s}=\mathbb{S}^{n} \times \mathbb{S}^{n}$ и $Q_{h}=$ $\mathbb{H}^{n} \times \mathbb{H}^{n}$ и соответствуюшие им гамильтонианы

$$
\widehat{H}_{s, h}=-\frac{1}{2 m_{1}} \Delta_{1}-\frac{1}{2 m_{2}} \Delta_{2}+U\left(\rho^{s, h}\right) \equiv \widehat{H}_{0}^{s, h}+U\left(\rho^{s, h}\right),
$$

где $\Delta_{1}$ и $\Delta_{2}$ - операторы Лапласа-Бельтрами на пространствах $\mathbb{S}^{n}$ или $\mathbb{H}^{n}$, соответствующие первой и второй частицам, $U$ - центральный потенциал.

В соответствии с обшей концепцией квантовой механики [15], оператор $\widehat{H}_{s}\left(\widehat{H}_{h}\right)$ должен быть определен на подходящем всюду плотном подпространстве пространства $\mathcal{L}^{2}\left(Q_{s, h}, d \mu_{s, h}\right)$ квадратично-интегрируемых на $Q_{s}\left(Q_{h}\right)$ функций. Это подпространство выбирается так, чтобы оператор был самосопряженным, причем соответствующая мера $d \mu_{s}\left(d \mu_{h}\right)$ есть произведение двух экземпляров инвариантной относительно группы $\mathrm{SO}(n+1)(\mathrm{SO}(1, n))$ меры на пространстве $\mathbb{S}^{n}\left(\mathbb{H}^{n}\right)$.

Представим гамильтониан $\widehat{H}_{s, h}$ через радиальный дифференциальный оператор и генераторы группы изометрий. Для этого достаточно найти такое выражение для свободного гамильтониана. Напомним $[16,17]$, что оператор Лапласа-Бельтрами $\Delta$ на пространствах $\mathbb{S}^{n}$ и $\mathbb{H}^{n}$ является самосопряженным оператором с областями определения

$$
\begin{aligned}
& W_{s}^{2,2}:=\left\{\phi \in \mathcal{L}^{2}\left(\mathbb{S}^{n}, d \mu_{s}\right) \mid \Delta \phi \in \mathcal{L}^{2}\left(\mathbb{S}^{n}, d \mu_{s}\right)\right\} \\
& W_{h}^{2,2}:=\left\{\phi \in \mathcal{L}^{2}\left(\mathbb{H}^{n}, d \mu_{h}\right) \mid \Delta \phi \in \mathcal{L}^{2}\left(\mathbb{H}^{n}, d \mu_{h}\right)\right\}
\end{aligned}
$$

Действие оператора $\Delta$ при этом понимается в смысле обобщенных функций. Областью существенной самосопряженности для оператора $\Delta$ на $\mathbb{S}^{n}$ является пространство гладких функций $C^{\infty}\left(\mathbb{S}^{n}\right)$, а для оператора $\Delta$ на $\mathbb{H}^{n}$ - пространство финитных гладких функций $C_{0}^{\infty}\left(\mathbb{H}^{n}\right)$. Отсюда ясно, что свободный гамильтониан $\widehat{H}_{0}^{s, h}$ самосопряжен на произведении $W_{s, h}:=W_{s, h}^{2,2} \otimes W_{s, h}^{2,2}$ двух экземпляров пространств $W_{s, h}^{2,2}$, отвечающих первой и второй частицам. 
Пусть подмногообразия $F_{r}^{s, h}$ пространств $Q_{s, h}$ отвечают постоянному значению $r$ функции $\operatorname{tg}\left(\rho^{s} / 2 R\right)$ для пространства $Q_{s}$ и функции th $\left(\rho^{h} / 2 R\right)$ для пространства $Q_{h}$. Подмногообразия $F_{0}^{s}, F_{\infty}^{s}, F_{0}^{h}$ диффеоморфны $\mathbb{S}^{n}$ и $\mathbb{H}^{n}$ (значение $r=\infty$ отвечает диаметрально противоположным точкам на сфере $\left.\mathbb{S}^{n}\right)$. При $0<r<\infty$ подмногообразие $F_{r}^{s}$ есть однородное риманово пространство группы $\mathrm{SO}(n+1)$ со стационарной подгруппой $K=\mathrm{SO}(n-1)$. При $0<r<1$ подмногообразие $F_{r}^{h}$ есть однородное риманово пространство группы $\mathrm{SO}(1, n)$ со стационарной подгруппой $K$.

С точностью до множества нулевой меры $Q_{s}=\mathbb{R}_{+} \times(\mathrm{SO}(n+1) / K)$, где $\mathbb{R}_{+}=(0, \infty)$, и $Q_{h}=I \times(\mathrm{SO}(1, n) / K)$, где $I=(0,1)$. Операторы $\widehat{H}_{0}^{s, h}$ являются операторами Лапласа-Бельтрами для метрики $\tilde{g}_{s, h}=2 m_{1} g_{s, h}^{(1)}+2 m_{2} g_{s, h}^{(2)}$ на $Q_{s, h}$, где $g_{s, h}^{(1)}$ и $g_{s, h}^{(2)}$ суть метрики (1) и (3) на разных экземплярах $\mathbb{S}^{n}$ и $\mathbb{H}^{n}$, соответствуюшие частицам 1 и 2.

3.1. Гамильтониан на сфере $\mathbb{S}^{n}$. Выбрав точку $\mathbf{x}_{0} \in F_{r}$, мы можем отождествить слой $F_{r}$ с факторпространством $\mathrm{SO}(n+1) / \mathrm{SO}(n-1)$ в соответствии с формулой $\mathbf{x}=g K \mathbf{x}_{0}$, где $g K$ - левый смежный класс элемента $g$ в группе $\mathrm{SO}(n+1)$. Пусть $\left(r, y_{1}, \ldots, y_{2 n-1}\right)$ - локальные координаты в окрестности $W$ точки $\mathbf{x}_{0} \in Q_{s}$ такие, что $\left(y_{1}, \ldots, y_{2 n-1}\right)$ - координаты в любом непустом открытом подмножестве $W \cap F_{r}$ пространства $Q_{s}$. Тогда метрика $\tilde{g}_{s}$ в $W$ принимает вид

$$
\tilde{g}_{s}=g_{r r}(r) d r^{2}+\sum_{i, j=1}^{2 n-1} g_{i j}\left(r, y_{1}, \ldots, y_{2 n-1}\right) d y_{i} d y_{j}
$$

Второе слагаемое в этой формуле есть ограничение некоторой метрики $g_{f}$ со слоя $F_{r}$ на множество $U \cap F_{r}$. Используя стандартное выражение оператора Лапласа-Бельтрами через локальные координаты, мы получаем

$$
\Delta_{\tilde{g}_{s}}=\left(g_{r r} \operatorname{det} g_{i j}\right)^{-\frac{1}{2}} \frac{\partial}{\partial r}\left(\sqrt{g^{r r} \operatorname{det} g_{i j}} \frac{\partial}{\partial r}\right)+\Delta_{g_{f}} .
$$

Нам необходимо выразить оператор $\Delta_{g_{f}}$ на $F_{r}$ через генераторы группы Ли $\mathrm{SO}(n+1)$. С этой целью поднимем его на группу $\mathrm{SO}(n+1)$, воспользовавшись следуюшей конструкцией [18]. Пусть $\Gamma$ - группа Ли, а $\Gamma_{0}$ - ее компактная подгруппа. Группа $Г$ действует слева на однородном пространстве $\Gamma / \Gamma_{0}$. Левоинвариантные дифференциальные операторы на пространстве $Г / \Gamma_{0}$ могут быть представлены левоинвариантными операторами на группе $\Gamma$, которые также инвариантны относительно правого действия группы $\Gamma_{0}$. Это представление однозначно с точностью до операторных слагаемых, обрашающихся в нуль при действии на правоинвариантные относительно $\Gamma_{0}$ функции.

Действительно, функции на пространстве $\Gamma / \Gamma_{0}$ находятся во взаимно однозначном соответствии с функциями на группе $Г$, которые инвариантны относительно правого действия подгруппы $\Gamma_{0}$. Это соответствие задается формулой $\lambda: f \rightarrow \tilde{f}:=f \circ \pi$, где $\pi-$ каноническая проекция $\Gamma \rightarrow \Gamma / \Gamma_{0}, f$ - функция на пространстве $\Gamma / \Gamma_{0}$. Пусть $D-$ левоинвариантный относительно группы $\Gamma$ дифференциальный оператор на $\Gamma$, являющийся также правоинвариантным относительно $\Gamma_{0}$, а $f$ - гладкая функция на пространстве 
$\Gamma / \Gamma_{0}$. Тогда формула $\widetilde{D_{u} f}=D \tilde{f}$, где $D_{u}-$ дифференциальный оператор на пространстве $\Gamma / \Gamma_{0}$, инвариантный относительно левого действия $\Gamma$, задает упомянутое соответствие $D \rightarrow D_{u}$

Пусть $e_{1}, \ldots, e_{N}$ - базис алгебры Ли группы $\Gamma, N:=\operatorname{dim} \Gamma$, а $L_{\gamma}$ и $R_{\gamma}$ обозначают левый и правый сдвиги на элемент $\gamma$. Алгебра левоинвариантных дифференциальных операторов на группе $Г$ порождена над полем $\mathbb{R}$ левоинвариантными векторными полями $e_{1}^{l}, \ldots, e_{N}^{l}$, где $e_{i}^{l}(\gamma)=d L_{\gamma}\left(e_{i}\right), \gamma \in \Gamma, i=1, \ldots, N[18]$.

Пусть теперь $\Gamma=\mathrm{SO}(n+1), \Gamma_{0}=K, e_{i}^{r}(\gamma)=d R_{\gamma}\left(e_{i}\right), i=1, \ldots, N, N=(n+1)(n+$ $2) / 2, \mathbf{x}_{0}=(r_{1}, \underbrace{0, \ldots, 0}_{n-1}, r_{2}, \underbrace{0, \ldots, 0}_{n-1}) \in \mathbb{S}^{n} \times \mathbb{S}^{n}$, где

$$
r_{1}=\operatorname{tg}\left(\frac{m_{2}}{m_{1}+m_{2}} \operatorname{arctg} r\right), \quad r_{2}=-\operatorname{tg}\left(\frac{m_{1}}{m_{1}+m_{2}} \operatorname{arctg} r\right) .
$$

Множество полей Киллинга $X_{i j}^{s}, Y_{i}^{s}, i, j=1, \ldots, n$, на пространстве $\mathbb{S}^{n} \times \mathbb{S}^{n}$, соответствующих (2), совпадает (с точностью до перестановки) с множеством

$$
\left\{\tilde{e}_{i}^{r}\left(\gamma x_{0}\right)=\left.\frac{d}{d \tau}\right|_{\tau=0} \exp \left(\tau e_{i}\right) \gamma \mathbf{x}_{0}\right\}_{i=1}^{N}, \quad \mathbf{x}_{0}=\mathbf{x}_{0}(r), \quad 0<r<\infty,
$$

при соответствуюшем выборе базиса $e_{1}^{l}, \ldots, e_{N}^{l}$. Пусть $\Delta_{f}$ - дифференциальный оператор второго порядка на группе $\Gamma$, так что $\left(\Delta_{f}\right)_{u}=\Delta_{g_{f}}$. Тогда он левоинвариантен и может быть выражен в следуюшей форме ${ }^{1)}$ :

$$
\left.\Delta_{f}\right|_{\gamma}=\sum_{i, j=1}^{N} c^{i j} e_{i}^{l}(\gamma) e_{j}^{l}(\gamma)+\sum_{i=1}^{N} c^{i} e_{i}^{l}(\gamma)
$$

где $c^{i j}, c^{i}$ - постоянные на слое $F_{r}$. Пусть е - единица группы Г. Очевидно, что $e_{i}^{r}(\mathrm{e})=$ $e_{i}^{l}(\mathrm{e}), i=1, \ldots, N$, и

$$
\left.\Delta_{f}\right|_{\mathrm{e}}=\sum_{i, j=1}^{N} c^{i j} e_{i}^{r}(\mathrm{e}) e_{j}^{r}(\mathrm{e})+\sum_{i=1}^{N} c^{i} e_{i}^{r}(\mathrm{e})
$$

Это значит, что

$$
\left.\Delta_{g_{f}}\right|_{\mathbf{x}_{0}}=\sum_{i, j=1}^{N} c^{i j} \tilde{e}_{i}^{r}\left(x_{0}\right) \tilde{e}_{j}^{r}\left(x_{0}\right)+\sum_{i=1}^{N} c^{i} \tilde{e}_{i}^{r}\left(x_{0}\right)=:\left.\Delta_{g_{f}}^{(2)}\right|_{\mathbf{x}_{0}}+\left.\Delta_{g_{f}}^{(1)}\right|_{\mathbf{x}_{0}} .
$$

Коэффициенты $c^{i j}$ могут быть найдены следуюшим образом. Упорядоченное множество векторов $\left\{Y_{1}^{s}\left(\mathbf{x}_{0}\right), \ldots, Y_{n}^{s}\left(\mathbf{x}_{0}\right), X_{12}^{s}\left(\mathbf{x}_{0}\right), \ldots, X_{1 n}^{s}\left(\mathbf{x}_{0}\right)\right\}$ может рассматриваться

\footnotetext{
1) Здесь мы отождествляем левоинвариантные векторные поля на $Г$ и элементы $T_{\mathrm{e}} \Gamma$.
} 
как базис в пространстве $T_{\mathbf{x}_{0}} F_{r}$. Пусть $\left\{Y^{1}, \ldots, Y^{n}, X^{2}, \ldots, X^{n}\right\}$ - дуальный базис. Тогда

$\left.g_{f}\right|_{\mathbf{x}_{0}}=a Y^{1} \otimes Y^{1}+\sum_{i=2}^{n}\left[Y^{1} \otimes\left(\alpha_{i} Y^{i}+\beta_{i} X^{i}\right)+\sum_{j=2}^{n}\left(\alpha_{i j} Y^{i} \otimes Y^{j}+\beta_{i j} X^{i} \otimes X^{j}+\gamma_{i j} Y^{i} \otimes X^{j}\right)\right]$,

где

$$
\begin{aligned}
a & =\left.\tilde{g}\right|_{\mathbf{x}_{0}}\left(Y_{1}^{s}\left(\mathbf{x}_{0}\right), Y_{1}^{s}\left(\mathbf{x}_{0}\right)\right)=2 R^{2}\left(m_{1}+m_{2}\right), \\
\alpha_{i} & =\left.\tilde{g}\right|_{\mathbf{x}_{0}}\left(Y_{1}^{s}\left(\mathbf{x}_{0}\right), Y_{i}^{s}\left(\mathbf{x}_{0}\right)\right)=0, \\
\beta_{i} & =\left.\tilde{g}\right|_{\mathbf{x}_{0}}\left(Y_{1}^{s}\left(\mathbf{x}_{0}\right), X_{1 i}^{s}\left(\mathbf{x}_{0}\right)\right)=0, \\
\alpha_{i j} & =\left.\tilde{g}\right|_{\mathbf{x}_{0}}\left(Y_{i}^{s}\left(\mathbf{x}_{0}\right), Y_{j}^{s}\left(\mathbf{x}_{0}\right)\right)=2 R^{2} \sum_{k=1}^{2} \frac{m_{k}\left(1-r_{k}^{2}\right)^{2}}{\left(1+r_{k}^{2}\right)^{2}} \delta_{i j}, \\
\beta_{i j} & =\left.\tilde{g}\right|_{\mathbf{x}_{0}}\left(X_{1 i}^{s}\left(\mathbf{x}_{0}\right), X_{1 j}^{s}\left(\mathbf{x}_{0}\right)\right)=8 R^{2} \sum_{k=1}^{2} \frac{m_{k} r_{k}^{2}}{\left(1+r_{k}^{2}\right)^{2}} \delta_{i j}, \\
\gamma_{i j} & =\left.\tilde{g}\right|_{\mathbf{x}_{0}}\left(Y_{i}^{s}\left(\mathbf{x}_{0}\right), X_{1 j}^{s}\left(\mathbf{x}_{0}\right)\right)=4 R^{2} \sum_{k=1}^{2} \frac{m_{k} r_{k}\left(1-r_{k}^{2}\right)}{\left(1+r_{k}^{2}\right)^{2}} \delta_{i j}, \quad i, j=2, \ldots, n .
\end{aligned}
$$

Следовательно, мы получаем

$$
\left.\Delta_{g_{f}}^{(2)}\right|_{\mathbf{x}_{0}}=\frac{1}{a}\left(Y_{1}^{s}\left(\mathbf{x}_{0}\right)\right)^{2}+\sum_{i=2}^{n}\left[A_{s}\left(X_{1 i}^{s}\left(\mathbf{x}_{0}\right)\right)^{2}+C_{s}\left(Y_{i}^{s}\left(\mathbf{x}_{0}\right)\right)^{2}+B_{s}\left\{X_{1 i}^{s}\left(\mathbf{x}_{0}\right), Y_{i}^{s}\left(\mathbf{x}_{0}\right)\right\}\right]
$$

где $\{\cdot, \cdot\}$ обозначает антикоммутатор,

$$
\begin{aligned}
A_{s} & =\frac{m_{1}\left(1-r_{1}^{2}\right)^{2}\left(1+r_{2}^{2}\right)^{2}+m_{2}\left(1+r_{1}^{2}\right)^{2}\left(1-r_{2}^{2}\right)^{2}}{8 R^{2} m_{1} m_{2}\left(r_{1}-r_{2}\right)^{2}\left(1+r_{1} r_{2}\right)^{2}} \\
B_{s} & =-\frac{m_{1} r_{1}\left(1-r_{1}^{2}\right)\left(1+r_{2}^{2}\right)^{2}+m_{2} r_{2}\left(1-r_{2}^{2}\right)\left(1+r_{1}^{2}\right)^{2}}{4 R^{2} m_{1} m_{2}\left(r_{1}-r_{2}\right)^{2}\left(1+r_{1} r_{2}\right)^{2}} \\
C_{s} & =\frac{m_{1} r_{1}^{2}\left(1+r_{2}^{2}\right)^{2}+m_{2} r_{2}^{2}\left(1+r_{1}^{2}\right)^{2}}{2 R^{2} m_{1} m_{2}\left(r_{1}-r_{2}\right)^{2}\left(1+r_{1} r_{2}\right)^{2}} .
\end{aligned}
$$

Функции $A_{s}, B_{s}, C_{s}$ можно выразить через координату $r$ :

$$
\begin{gathered}
A_{s}(r)=\frac{1}{2 R^{2}}\left(\frac{\left(1+r^{2}\right)^{2}}{8 m r^{2}}+\frac{1-r^{4}}{8 m r^{2}} \cos \zeta+\frac{1+r^{2}}{4 m_{1} m_{2} r}\left(m_{1}-m_{2}\right) \sin \zeta\right), \\
B_{s}(r)=\frac{1}{2 R^{2}}\left(\frac{m_{2}-m_{1}}{m_{1} m_{2} r}\left(1+r^{2}\right) \cos \zeta+\frac{1-r^{4}}{2 m r^{2}} \sin \zeta\right), \\
C_{s}(r)=\frac{1}{2 R^{2}}\left(\frac{\left(1+r^{2}\right)^{2}}{8 m r^{2}}-\frac{1-r^{4}}{8 m r^{2}} \cos \zeta-\frac{1+r^{2}}{4 m_{1} m_{2} r}\left(m_{1}-m_{2}\right) \sin \zeta\right), \\
\zeta=2 \frac{m_{1}-m_{2}}{m_{1}+m_{2}} \operatorname{arctg} r, \quad m=\frac{m_{1} m_{2}}{m_{1}+m_{2}} .
\end{gathered}
$$


Операторы $\left.\Delta_{g_{f}}\right|_{\mathbf{x}_{0}}$ и $\left.\Delta_{g_{f}}^{(2)}\right|_{\mathbf{x}_{0}}(10)$ инвариантны относительно отражений сферы $\mathbb{S}^{n}$ $T_{k}: x_{k} \rightarrow-x_{k}, x_{j} \rightarrow x_{j}, j \neq k$, поэтому оператор $\left.\Delta_{g_{f}}^{(1)}\right|_{\mathbf{x}_{0}}$ также инвариантен относительно этих преобразований. Но для оператора первого порядка с постоянными коэффициентами это возможно, только если он равен нулю, поэтому $c^{i}=0, i=1, \ldots, N$.

Если обозначить левоинвариантные векторные поля на группе $\mathrm{SO}(n+1)$, соответствуюшие векторам $Y_{1}^{s}\left(\mathbf{x}_{0}\right), X_{1 i}^{s}\left(\mathbf{x}_{0}\right), Y_{i}^{s}\left(\mathbf{x}_{0}\right), i=2, \ldots, n$, через $Y_{1}^{s, l}, X_{i}^{s, l}, Y_{i}^{s, l}$, то получим

$$
\Delta_{f}=\frac{1}{a}\left(Y_{1}^{s, l}\right)^{2}+\sum_{i=2}^{n}\left[A_{s}\left(X_{i}^{s, l}\right)^{2}+C_{s}\left(Y_{i}^{s, l}\right)^{2}+\frac{1}{4} B_{s}\left\{X_{i}^{s, l}, Y_{i}^{s, l}\right\}\right] .
$$

Таким образом, мы нашли оператор $\Delta_{f}$ с точностью до слагаемых, аннулируемых правоинвариантными относительно подгруппы $\Gamma_{0}$ функциями. Можно проверить прямыми вычислениями, что этот оператор правоинвариантен относительно $K$.

Найдем теперь первое слагаемое в выражении (6) для оператора $\Delta_{\tilde{g}}$. В точке $\mathbf{x}_{0}$ мы получаем

поэтому

$$
\frac{\partial}{\partial r}=\frac{m_{2}}{m_{1}+m_{2}} \frac{1+r_{1}^{2}}{1+r^{2}} \frac{\partial}{\partial r_{1}}-\frac{m_{1}}{m_{1}+m_{2}} \frac{1+r_{2}^{2}}{1+r^{2}} \frac{\partial}{\partial r_{2}},
$$

$$
g_{r r}=\tilde{g}\left(\frac{\partial}{\partial r}, \frac{\partial}{\partial r}\right)=\frac{8 R^{2} m_{1} m_{2}}{\left(m_{1}+m_{2}\right)\left(1+r^{2}\right)^{2}} .
$$

Принимая во внимание формулы (9), получим

$$
\Delta_{\tilde{g}}=\frac{\left(1+r^{2}\right)^{n}}{8 m R^{2} r^{n-1}} \frac{\partial}{\partial r}\left(\frac{r^{n-1}}{\left(1+r^{2}\right)^{n-2}} \frac{\partial}{\partial r}\right)+\Delta_{g_{f}} .
$$

Первое слагаемое является радиальной частью одночастичного гамильтониана, соответствуюшего массе $m$.

Явные вычисления дают для меры $d \mu_{s}$, соответствующей метрике $\tilde{g}$ на пространстве $Q_{s}$ в точке $\mathbf{x}_{0}$, следующее выражение (с точностью до постоянного множителя):

$$
\left.d \mu_{s}\right|_{\mathbf{x}_{0}}=\frac{r^{n-1}}{\left(1+r^{2}\right)^{n}} d r \wedge Y^{1} \wedge \ldots \wedge Y^{n} \wedge X^{2} \wedge \ldots \wedge X^{n} .
$$

Мера $d \mu_{s}$ инвариантна относительно левого действия группы $\mathrm{SO}(n+1)$, и, следовательно, она может быть представлена в виде $d \mu_{s}=d \nu_{s} \otimes d \mu_{f}$, где $d \nu_{s}=r^{n-1} d r /\left(1+r^{2}\right)^{n}$ мера на множестве $\mathbb{R}_{+}=(0, \infty)$ такая же, как и в одночастичном случае, а $d \mu_{f}$ - левоинвариантная относительно группы $\mathrm{SO}(n+1)$ мера на пространстве $\mathrm{SO}(n+1) / K$.

На любой группе Ли сушествует единственная (с точностью до постоянного множителя) левоинвариантная (или правоинвариантная) мера (мера Хаара [19]). Для рассматриваемых групा $\mathrm{SO}(n+1)$ и $\mathrm{SO}(1, n)$ такие меры являются двусторонне инвариантными. Поэтому существует единственная двусторонне инвариантная мера $d \eta_{s}$ на группе $\mathrm{SO}(n+1)$ такая, что интеграл от любой интегрируемой функции $f$ на пространстве $\mathrm{SO}(n+1) / K$ по мере $d \mu_{f}$ равен интегралу от функции $\tilde{f}$ на группе $\mathrm{SO}(n+1)$ по мере $d \eta_{s}$.

Для подгруппы $\Gamma_{0}$ группы Ли $Г$ обозначим $\mathcal{L}^{2}\left(\Gamma, \Gamma_{0}, d \eta\right)$ пространство квадратично-интегрируемых функций на группе $\Gamma$ (относительно меры $d \eta$ на $\Gamma$ ), которые инвариантны относительно правого действия $\Gamma_{0}$. 
ТЕОРема 1. Свободный квантовый гамильтониан системы двух частии на сфере $\mathbb{S}^{n}$ можно рассматривать как самосопряжсенньй дифференииальньй оператор (на многообразии $\left.\widetilde{Q}_{s}=\mathbb{R}_{+} \times \mathrm{SO}(n+1)\right)$ в пространстве $\mathcal{H}_{s}$

$$
\widehat{H}_{0}^{s}=-\frac{\left(1+r^{2}\right)^{n}}{8 m R^{2} r^{n-1}} \frac{\partial}{\partial r}\left(\frac{r^{n-1}}{\left(1+r^{2}\right)^{n-2}} \frac{\partial}{\partial r}\right)-\Delta_{f}
$$

с областью определения

$$
D_{s}:=D_{s}^{(1)} \otimes D_{s}^{(2)} \subset \mathcal{H}_{s}:=\mathcal{L}^{2}\left(\mathbb{R}_{+}, d \nu_{s}\right) \otimes \mathcal{L}^{2}\left(\mathrm{SO}(n+1), K, d \eta_{s}\right),
$$

$2 \partial e$

$$
\begin{aligned}
& D_{s}^{(1)}:=\left\{\phi \in \mathcal{L}^{2}\left(\mathbb{R}_{+}, d \nu_{s}\right) \mid \Delta_{s}^{(1)} \phi \in \mathcal{L}^{2}\left(\mathbb{R}_{+}, d \nu_{s}\right)\right\}, \\
& D_{s}^{(2)}:=\left\{\phi \in \mathcal{L}^{2}\left(\mathrm{SO}(n+1), K, d \eta_{s}\right) \mid \Delta_{f} \phi \in \mathcal{L}^{2}\left(\mathrm{SO}(n+1), K, d \eta_{s}\right)\right\}, \\
& \Delta_{s}^{(1)}:=-\frac{\left(1+r^{2}\right)^{n}}{r^{n-1}} \frac{\partial}{\partial r}\left(\frac{r^{n-1}}{\left(1+r^{2}\right)^{n-2}} \frac{\partial}{\partial r}\right),
\end{aligned}
$$

подгруппа $K$ изоморфна группе $\mathrm{SO}(n-1)$, а $d \eta_{s}$ - однозначно определенная (с точностью до постоянного множителя) двусторонне инвариантная мера на группе $\mathrm{SO}(n+1)$. Это означает, что существует изометрия исходного функиионального пространства $\mathcal{L}^{2}\left(Q_{s}, d \mu_{s}\right)$ на $\mathcal{H}_{s}$, которая порождает изоморфизм гамильтонианов. Пространство $D_{s}$ всюду плотно в $\mathcal{H}_{s}$.

ДокАЗАТЕльство. Выражение (6) является формой записи гамильтониана $\widehat{H}_{0}^{s}$ в системе координат, связанной с представлением $Q_{s}$ в виде прямого произведения $\mathbb{R}_{+} \times$ $\mathrm{SO}(n+1) / \mathrm{SO}(n-1)$ с точностью до множества нулевой меры $F_{0}^{s} \cup F_{\infty}^{s}$, которое несущественно для изучения суммируемых по этой мере функций, поэтому $\mathcal{L}^{2}\left(Q_{s}, d \mu_{s}\right)=$ $\mathcal{L}^{2}\left(\mathbb{R}_{+}, d \nu_{s}\right) \otimes \mathcal{L}^{2}\left(\mathrm{SO}(n+1) / \mathrm{SO}(n-1), d \mu_{f}\right)$. Изометрия $\lambda: f \rightarrow \tilde{f}$ пространств $\mathcal{L}^{2}\left(\mathrm{SO}(n+1) / \mathrm{SO}(n-1), d \mu_{f}\right)$ и $\mathcal{L}^{2}\left(\mathrm{SO}(n+1), \mathrm{SO}(n-1), d \eta_{s}\right)$ порождает изометрию $\mathrm{id} \otimes \lambda$ пространств $\mathcal{L}^{2}\left(\mathbb{R}_{+}, d \nu_{s}\right) \otimes \mathcal{L}^{2}\left(\mathrm{SO}(n+1) / \mathrm{SO}(n-1), d \mu_{f}\right)$ и $\mathcal{H}_{s}$. Изометрия id $\otimes \lambda$, как следует из проведенных вычислений, и переводит оператор (6) в оператор (13), при этом пространство $W_{s}$ переходит в пространство $D_{s}$

ЗАМЕчАНИЕ. В случае $n=2$ этот результат может быть получен при рассмотрении базиса левоинвариантных векторных полей на групе $\mathrm{SO}(n+1)=\mathrm{SO}(3)$ как подвижного репера на слое $F_{r}[14]$. В случае $n>2$ такой путь невозможен, поскольку действие группы $\mathrm{SO}(n+1)$ на слое $F_{r}$ не является свободным и левоинвариантные векторные поля на группе $\mathrm{SO}(n+1)$ не проецируются однозначно на пространство $\mathrm{SO}(n+1) / \mathrm{SO}(n-1)$. Подъем гамильтониана на группу симметрий позволяет получить его выражение через генераторы группы.

3.2. Гамильтониан на гиперболическом пространстве $\mathbb{H}^{n}$. Формальная замена $x_{j} \rightarrow i x_{j}, j=1, \ldots, n, r \rightarrow i r, R \rightarrow i R$ ( $i$ - мнимая единица) переводит объекты на сфере $\mathbb{S}^{n}$ в объекты на гиперболическом пространстве $\mathbb{H}^{n}$ (см. также [6]), поэтому 
мы получаем выражение для двухчастичного свободного гамильтониана на пространстве $\mathbb{H}^{n}$

$$
\begin{aligned}
\widehat{H}_{0}^{h}= & -\frac{\left(1-r^{2}\right)^{n}}{8 m R^{2} r^{n-1}} \frac{\partial}{\partial r}\left(\frac{r^{n-1}}{\left(1-r^{2}\right)^{n-2}} \frac{\partial}{\partial r}\right)-\frac{1}{a}\left(Y_{1}^{h, l}\right)^{2}- \\
& -\sum_{k=2}^{n}\left[A_{h}\left(X_{k}^{h, l}\right)^{2}-C_{h}\left(Y_{k}^{h, l}\right)^{2}+\frac{1}{4} B_{h}\left\{X_{k}^{h, l}, Y_{k}^{h, l}\right\}\right],
\end{aligned}
$$

где векторные поля $X_{k}^{h, l}, Y_{k}^{h, l}$ соответствуют полям (4) так же, как поля $X_{k}^{s, l}, Y_{k}^{s, l}$ соответствуют полям (2),

$$
\begin{gathered}
A_{h}(r)=\frac{1}{2 R^{2}}\left(\frac{\left(1-r^{2}\right)^{2}}{8 m r^{2}}+\frac{1-r^{4}}{8 m r^{2}} \operatorname{ch} \zeta-\frac{1-r^{2}}{4 m_{1} m_{2} r}\left(m_{1}-m_{2}\right) \operatorname{sh} \zeta\right), \\
B_{h}(r)=\frac{1}{2 R^{2}}\left(\frac{m_{2}-m_{1}}{m_{1} m_{2} r}\left(1-r^{2}\right) \operatorname{ch} \zeta+\frac{1-r^{4}}{2 m r^{2}} \operatorname{sh} \zeta\right), \\
C_{h}(r)=\frac{1}{2 R^{2}}\left(\frac{\left(1-r^{2}\right)^{2}}{8 m r^{2}}-\frac{1-r^{4}}{8 m r^{2}} \operatorname{ch} \zeta+\frac{1-r^{2}}{4 m_{1} m_{2} r}\left(m_{1}-m_{2}\right) \operatorname{sh} \zeta\right), \\
\zeta=2 \frac{m_{1}-m_{2}}{m_{1}+m_{2}} \operatorname{arcth} r .
\end{gathered}
$$

Теорема 2. Свободный квантовый двухчастичный гамильтониан на гиперболическом пространстве $\mathbb{H}^{n}$ можно рассматривать как самосопряженный дифференциальныц й оператор (14) (на многообразии $\left.\widetilde{Q}_{h}=I \times \mathrm{SO}(1, n)\right)$ в пространстве $\mathcal{H}_{h}$ с областью определения

$$
D_{h}:=D_{h}^{(1)} \otimes D_{h}^{(2)} \subset \mathcal{H}_{h}:=\mathcal{L}^{2}\left(\mathbb{R}_{+}, d \nu_{h}\right) \otimes \mathcal{L}^{2}\left(\operatorname{SO}(1, n), K, d \eta_{h}\right),
$$

ฉде

$$
\begin{aligned}
D_{h}^{(1)} & :=\left\{\phi \in \mathcal{L}^{2}\left(\mathbb{R}_{+}, d \nu_{h}\right) \mid \Delta_{h}^{(1)} \phi \in \mathcal{L}^{2}\left(\mathbb{R}_{+}, d \nu_{h}\right)\right\}, \\
D_{h}^{(2)} & :=\left\{\phi \in \mathcal{L}^{2}\left(\operatorname{SO}(1, n), K, d \eta_{h}\right) \mid \Delta_{h} \phi \in \mathcal{L}^{2}\left(\mathrm{SO}(1, n), K, d \eta_{h}\right)\right\}, \\
\Delta_{h}^{(1)} & :=-\frac{\left(1-r^{2}\right)^{n}}{r^{n-1}} \frac{\partial}{\partial r}\left(\frac{r^{n-1}}{\left(1-r^{2}\right)^{n-2}} \frac{\partial}{\partial r}\right), \quad d \nu_{h}=\frac{r^{n-1} d r}{\left(1-r^{2}\right)^{n}}, \\
\Delta_{h} & :=-\frac{1}{a}\left(Y_{1}^{h, l}\right)^{2}-\sum_{k=2}^{n}\left[A_{h}\left(X_{k}^{h, l}\right)^{2}-C_{h}\left(Y_{k}^{h, l}\right)^{2}+\frac{1}{4} B_{h}\left\{X_{k}^{h, l}, Y_{k}^{h, l}\right\}\right],
\end{aligned}
$$

a $d \eta_{h}$ - единственная (с точностью до постоянного множителя) двусторонне инвариантная мера на группе $\mathrm{SO}(1, n)$.

ДоКАЗАТЕЛЬСТВО аналогично доказательству теоремы 1. 


\section{4. РЕДУКЦИЯ ГАМИЛЬТОНОВОЙ ДИНАМИЧЕСКОЙ СИСТЕМЫ НА КОКАСАТЕЛЬНОМ РАССЛОЕНИИ ОДНОРОДНОГО ПРОСТРАНСТВА}

Пусть $\Gamma$ - группа Ли с алгеброй $g, \Gamma_{0}-$ подгруппа группы $\Gamma$ с алгеброй $g_{0} \subset g$, действующая на $\Gamma$ справа. Пусть $M=T^{*} \Gamma_{1}$ - кокасательное расслоение однородного пространства $\Gamma_{1}=\Gamma / \Gamma_{0}$, наделенное стандартной симплектической структурой. Стандартное левое действие группы Г на $M$ является пуассоновым [13]. Пусть $\Phi: M \rightarrow g^{*}-$ соответствуюшее отображение момента, а $H-\Gamma$-инвариантная функция на $M$. Рассмотрим метод редукции Марсдена-Вейнстайна применительно к гамильтоновой динамической системе с функцией $H$ на многообразии $M$. Хорошо известно [13], что для $\Gamma_{0}=\{\mathrm{e}\}$ приведенное фазовое пространство симплектоморфно орбите группы Г в кокасательном расслоении, снабженной формой Кириллова. Следуюшая конструкция обобшает данное утверждение.

Пусть $O_{\beta_{0}}$ - орбита коприсоединенного действия группы $\Gamma$ на $g^{*}$, содержашая точку $\beta_{0} \in g^{*}$, и $O_{\beta_{0}}^{\prime}:=\left\{\beta \in O_{\beta_{0}}|\beta|_{g_{0}}=0\right\}$. Очевидно, что $\operatorname{Ad}_{\Gamma_{0}}^{*} O_{\beta_{0}}^{\prime}=O_{\beta_{0}}^{\prime}$. Пусть $\widetilde{O}_{\beta_{0}}=$ $O_{\beta_{0}}^{\prime} / \operatorname{Ad}_{\Gamma_{0}}^{*}$ и $\pi: O_{\beta_{0}}^{\prime} \rightarrow \widetilde{O}_{\beta_{0}}-$ каноническая проекция. Пусть $\omega$ - ограничение формы Кириллова на $O_{\beta_{0}}^{\prime}$. Это означает, что если элементы $X, Y \in T_{\beta} O_{\beta_{0}}^{\prime}, \beta \in O_{\beta_{0}}^{\prime}$, имеют вид

$$
X=\left.\frac{d}{d t}\right|_{t=0} \operatorname{Ad}_{\exp \left(t X^{\prime}\right)}^{*} \beta, \quad Y=\left.\frac{d}{d t}\right|_{t=0} \operatorname{Ad}_{\exp \left(t Y^{\prime}\right)}^{*} \beta, \quad X^{\prime}, Y^{\prime} \in g,
$$

то $\omega(X, Y)=\beta\left(\left[X^{\prime}, Y^{\prime}\right]\right)$. Поскольку $\left.\operatorname{Ad}_{\exp \left(t X^{\prime}\right)}^{*} \beta\right|_{g_{0}}=0$, то

$$
\beta\left(\left[X^{\prime}, Y_{0}^{\prime}\right]\right)=\left.\frac{d}{d t}\right|_{t=0} \operatorname{Ad}_{\exp \left(t X^{\prime}\right)}^{*} \beta\left(Y_{0}^{\prime}\right)=0
$$

для любого элемента $Y_{0}^{\prime} \in g_{0}$. Это означает, что 2-форма $\widetilde{\omega}$ корректно определена на $T \widetilde{O}_{\beta_{0}}$ формулой $\widetilde{\omega}(\widetilde{X}, \widetilde{Y})=\omega\left(d \pi^{-1} \widetilde{X}, d \pi^{-1} \widetilde{Y}\right)$ для $\widetilde{X} \in T_{\pi \beta} \widetilde{O}_{\beta_{0}}, \widetilde{Y} \in T_{\pi \beta} \widetilde{O}_{\beta_{0}}$.

ТЕорема 3. Приведенное фазовое пространство $\widetilde{M}_{\beta_{0}}$, соответствующее значению отображения момента $\beta_{0}$, симплектоморфно симплектическому пространству $\left(\widetilde{O}_{\beta_{0}}, \widetilde{\omega}\right)$.

ДокаЗАтЕЛЬСтво. Рассмотрим точку $x \in M_{\beta_{0}}:=\Phi^{-1}\left(\beta_{0}\right) \subset M$ как орбиту $O_{x^{\prime}}$ некоторой точки $x^{\prime}=(\gamma, p) \in T^{*} \Gamma, \gamma \in \Gamma, p \in T_{\gamma}^{*} \Gamma$, относительно правого действия $\Gamma_{0}$ на $T^{*} \Gamma$. Чтобы избежать слишком громоздких обозначений, мы сохраним для левого и правого действий $(\gamma, p) \rightarrow\left(\gamma_{1} \gamma, L_{\gamma_{1}^{-1}}^{*} p\right)$ и $(\gamma, p) \rightarrow\left(\gamma \gamma_{1}, R_{\gamma_{1}^{-1}}^{*} p\right)$ элемента $\gamma_{1} \in \Gamma$ на $T^{*} \Gamma$ обозначения $L_{\gamma_{1}}$ и $R_{\gamma_{1}}$, соответственно. Согласно определению отображения момента, если

$$
X=\left.\frac{d}{d t}\right|_{t=0} L_{\exp \left(t X^{\prime}\right)} \gamma, \quad X^{\prime} \in g, \quad X \in T_{\gamma} \Gamma
$$

то $p(X)=\beta_{0}\left(X^{\prime}\right)$, т.е. $p=R_{\gamma^{-1}}^{*} \beta_{0}$. Если $X^{\prime} \in \operatorname{Ad}_{\gamma} g_{0}$, то $X \in d \pi_{1}\left(T_{x^{\prime}} O_{x^{\prime}}\right)$, где $\pi_{1}: T^{*} \Gamma \rightarrow \Gamma-$ стандартная проекция, $p(X)=0$. Следовательно, $\left.\operatorname{Ad}_{\gamma}^{*} \beta_{0}\right|_{g_{0}}=0$. 
Обозначим $O=\left\{x^{\prime}=(\gamma, p) \in T^{*} \Gamma\left|\operatorname{Ad}_{\gamma}^{*} \beta_{0}\right|_{g_{0}}=0, p=R_{\gamma^{-1}}^{*} \beta_{0}\right\}$. Пусть $\tau: O \rightarrow g^{*}=$ $T_{e}^{*} \Gamma$ - отображение, действуюшее по формуле $\tau(\gamma, p)=L_{\gamma}^{*} p$. Следуюшая диаграмма коммутативна [13]:

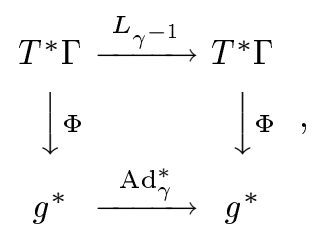

поэтому орбита стационарной подгруппы $\Gamma_{\beta_{0}}$ на $T^{*} \Gamma$ переводится отображением $\tau$ в одну точку. По определению множества $O$ справедливо $\tau(O)=O_{\beta_{0}}^{\prime}$, а элемент $(\gamma, p)$ переводится отображением $\tau$ в $\operatorname{Ad}_{\gamma}^{*} \beta_{0}$, поэтому элемент $R_{\gamma_{0}}(\gamma, p)$ переводится в $\operatorname{Ad}_{\gamma \gamma_{0}}^{*} \beta_{0}=$ $\operatorname{Ad}_{\gamma_{0}}^{*} \circ \operatorname{Ad}_{\gamma}^{*} \beta_{0}$. Следовательно, орбиты правого действия группы $\Gamma_{0}$ на $O$ переводятся в орбиты коприсоединенного действия группы $\Gamma_{0}$ на $O_{\beta_{0}}^{\prime}$. Таким образом, отображение $\tau$ индуцирует диффеоморфизм

$$
\phi: \widetilde{M}_{\beta_{0}}=\Gamma_{\beta_{0}} \backslash M_{\beta_{0}}=\Gamma_{\beta_{0}} \backslash\left(O / \Gamma_{0}\right) \rightarrow O_{\beta_{0}}^{\prime} / \operatorname{Ad}_{\Gamma_{0}}^{*}=\widetilde{O}_{\beta_{0}} .
$$

Остается доказать, что симплектическая форма $\widehat{\omega}$ на $\widetilde{M}_{\beta_{0}}$ под действием отображения $\phi$ переводится в форму - $\widetilde{\omega}$. Но этот факт следует из его справедливости для случая $\Gamma_{0}=\{$ е $\}$, возможности представления касательных векторов на пространстве $\widetilde{M}_{\beta_{0}}$ через касательные вектора на $O$ и коммутативности следуюшей диаграммы для любого $\gamma_{0} \in \Gamma_{0}$ :

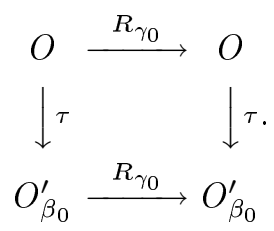

Форма $\widehat{\omega}$ симплектическая, поэтому мы получаем

СлЕдСтвиЕ. Форма $\widetilde{\omega}$ на $\widetilde{O}_{\beta_{0}}$ является симплектической, т.е. невырожденна и замкнута.

\section{5. РЕДУКЦИЯ КЛАССИЧЕСКОЙ ДИНАМИЧЕСКОЙ СИСТЕМЫ ДВУХ ТЕЛ}

В работе [6] было отмечено, что для пространств $\mathbb{H}^{n}$ и $\mathbb{S}^{n}, n \geqslant 3$, задача двух тел достигает максимальной общности при $n=3$, так как два элемента из пространства $T^{*} \mathbb{H}^{n}\left(T^{*} \mathbb{S}^{n}\right)$ всегда содержатся в некотором подпространстве $T^{*} \mathbb{H}^{3} \subset T^{*} \mathbb{H}^{n}\left(T^{*} \mathbb{S}^{3} \subset\right.$ $\left.T^{*} \mathbb{S}^{n}\right)$. Следовательно, две материальные точки с центральным взаимодействием останутся всегда в пространстве $\mathbb{H}^{3}\left(\mathbb{S}^{3}\right)$, поэтому мы рассмотрим случай $n=3$.

5.1. Задача двух частиц на сфере $\mathbb{S}^{3}$. Пусть теперь пространство $M=T^{*} Q_{s}$ снабжено стандартной симплектической структурой. Согласно п. 3.1 мы можем представить многообразие $M$ с точностью до множества нулевой меры, соответствуюшего значениям $r=0, \infty$, в виде

$$
M=T^{*} \mathbb{R}_{+} \times T^{*}(\mathrm{SO}(4) / \mathrm{SO}(2)) .
$$


Группа симметрий $\mathrm{SO}(4)$ действует только на второй сомножитель произведения (15), поэтому конструкция, описанная в разделе 4 , легко обобшается на данный случай. После редукции мы получаем вместо (15) пространство

$$
\widetilde{M}_{\beta_{0}}=T^{*} \mathbb{R}_{+} \times \widetilde{Q}_{\beta_{0}},
$$

где $\widetilde{Q}_{\beta_{0}}$ строится для пространств $\Gamma=\mathrm{SO}(4), \Gamma_{0}=\mathrm{SO}(2)$, как в разделе 4.

Введем конкретные координаты на пространстве $\widetilde{M}_{\beta_{0}}$ и используем формулу (13) для выражения функции Гамильтона через эти координаты. В случае $n=3$ векторные поля Киллинга (2) есть $X_{12}^{s}, X_{31}^{s}, X_{23}^{s}, Y_{1}^{s}, Y_{2}^{s}, Y_{3}^{s}$. Для простоты мы будем использовать эти обозначения также для базиса алгебры so(4) (без индекса “s”) в соответствии с (7). Пусть $X^{12}, X^{31}, X^{23}, Y^{1}, Y^{2}, Y^{3}$ - дуальный базис. Введем также другой базис на алгебре so(4):

$$
\begin{aligned}
& L_{1}=\frac{1}{2}\left(X_{23}^{s}+Y_{1}^{s}\right), \quad L_{2}=\frac{1}{2}\left(X_{31}^{s}+Y_{2}^{s}\right), \quad L_{3}=\frac{1}{2}\left(X_{12}^{s}+Y_{3}^{s}\right), \\
& G_{1}=\frac{1}{2}\left(X_{23}^{s}-Y_{1}^{s}\right), \quad G_{2}=\frac{1}{2}\left(X_{31}^{s}-Y_{2}^{s}\right), \quad G_{3}=\frac{1}{2}\left(X_{12}^{s}-Y_{3}^{s}\right) \text {. }
\end{aligned}
$$

Для этого базиса справедливы соотношения

$$
\left[L_{i}, L_{j}\right]=\sum_{k=1}^{3} \varepsilon_{i j k} L_{k}, \quad\left[G_{i}, G_{j}\right]=\sum_{k=1}^{3} \varepsilon_{i j k} G_{k}, \quad\left[L_{i}, G_{j}\right]=0, \quad i, j=1,2,3,
$$

где $\varepsilon_{i j k}$ - полностью антисимметричный тензор, $\varepsilon_{123}=1$. Этот базис соответствует разложению $\mathrm{so}(4)=\mathrm{so}(3) \oplus \mathrm{so}(3)$. Пусть

$$
\begin{array}{lll}
L^{1}=X^{23}+Y^{1}, & L^{2}=X^{31}+Y^{2}, & L^{3}=X^{12}+Y^{3} \\
G^{1}=X^{23}-Y^{1}, & G^{2}=X^{31}-Y^{2}, & G^{3}=X^{12}-Y^{3}
\end{array}
$$

- дуальный базис. Для произвольного элемента пространства so*(4) мы будем использовать обозначение

$$
\mathbf{p}=p_{1} X^{23}+p_{2} X^{31}+p_{3} X^{12}+p_{4} Y^{1}+p_{5} Y^{2}+p_{6} Y^{3}=\sum_{i=1}^{3}\left(u_{i} L^{i}+v_{i} G^{i}\right) .
$$

Стандартное соответствие между классическим и квантовым гамильтонианами и формулы (11) и (13) дают следующее выражение для классической функции Гамильтона:

$$
H_{s}=\frac{\left(1+r^{2}\right)^{2}}{8 m R^{2}} p_{r}^{2}+\frac{1}{a} p_{4}^{2}+A_{s}\left(p_{2}^{2}+p_{3}^{2}\right)+C_{s}\left(p_{5}^{2}+p_{6}^{2}\right)+\frac{1}{2} B_{s}\left(p_{3} p_{5}-p_{2} p_{6}\right)+U(r),
$$

где $p_{r}$ - импульс, сопряженньй координате $r$. После подстановки $p_{i}=u_{i}+v_{i}, p_{3+i}=$ $u_{i}-v_{i}, i=1,2,3$, мы получаем

$$
\begin{aligned}
H_{s}= & \frac{\left(1+r^{2}\right)^{2}}{8 m R^{2}} p_{r}^{2}+\frac{1}{a}\left(u_{1}-v_{1}\right)^{2}+A_{s}\left(\left(u_{2}+v_{2}\right)^{2}+\left(u_{3}+v_{3}\right)^{2}\right)+ \\
& +C_{s}\left(\left(u_{2}-v_{2}\right)^{2}+\left(u_{3}-v_{3}\right)^{2}\right)+B_{s}\left(u_{2} v_{3}-v_{2} u_{3}\right)+U(r) .
\end{aligned}
$$


Построим теперь канонически-сопряженные координаты на пространстве $\widetilde{O}_{\beta_{0}}$. Благодаря специальному выбору точки $\mathrm{x}_{0}$ на слое $F_{r}$ в п. 3.1, ее стационарная подгруппа $\mathrm{SO}(2)$ порождается элементом $X_{23}$. Хорошо известно, что орбиты коприсоединенного действия групшы $\mathrm{SO}(3)$ - это сферы, а форма Кириллова на них - площадь этих сфер. Поэтому орбита $O_{\beta_{0}}$ может быть представлена как множество элементов вида (17) таких, что координаты $u_{i}, v_{i}, i=1,2,3$, удовлетворяют соотношениям

$$
u_{1}^{2}+u_{2}^{2}+u_{3}^{2}=\mu^{2}, \quad v_{1}^{2}+v_{2}^{2}+v_{3}^{2}=\nu^{2},
$$

где $\mu, \nu$ - неотрицательные вешественные числа. Подмножество $O_{\beta_{0}}^{\prime} \subset O_{\beta_{0}}$ состоит из тех элементов $O_{\beta_{0}}$, которые аннулируются элементами $X_{23}$, поэтому для описания множества $O_{\beta_{0}}^{\prime}$ мы должны добавить к уравнениям (18) условие $p_{1}=u_{1}+v_{1}=0$.

Сначала рассмотрим случай $\mu, \nu>0$. Пусть $u, \psi, \chi-$ координаты на пространстве $O_{\beta_{0}}^{\prime}$, вводимые уравнениями

$$
\begin{array}{lll}
u_{1}=-v_{1}=u, & u_{2}=\sqrt{\mu^{2}-u^{2}} \sin \psi, & u_{3}=\sqrt{\mu^{2}-u^{2}} \cos \psi, \\
v_{2}=\sqrt{\nu^{2}-u^{2}} \sin \chi, & v_{3}=\sqrt{\nu^{2}-u^{2}} \cos \chi, & -\min \{\mu, \nu\} \leqslant u \leqslant \min \{\mu, \nu\} .
\end{array}
$$

Ограничение формы Кириллова с $O_{\beta_{0}}$ на $O_{\beta_{0}}^{\prime}$ имеет вид

$$
\begin{aligned}
\omega= & \frac{1}{\mu^{2}}\left(u_{1} d u_{2} \wedge d u_{3}+u_{2} d u_{3} \wedge d u_{1}+u_{3} d u_{1} \wedge d u_{2}\right)+ \\
& +\frac{1}{\nu^{2}}\left(v_{1} d v_{2} \wedge d v_{3}+v_{2} d v_{3} \wedge v_{1}+v_{3} d v_{1} \wedge d v_{2}\right)=d u \wedge d(\psi-\chi) .
\end{aligned}
$$

Коприсоединенное действие однопараметрической группы, соответствующей элементу $X_{23}$, на $O_{\beta_{0}}^{\prime}$ описывается формулами $u \rightarrow u, \psi \rightarrow \psi+\xi, \chi \rightarrow \chi+\xi, 0 \leqslant \xi<2 \pi$, поэтому $\phi=\psi-\chi, p_{\phi}=u$ - канонически-сопряженные координаты на $\widetilde{O}_{\beta_{0}}$. Фактически пространство $\widetilde{O}_{\beta_{0}}$ диффеоморфно двумерной сфере. Координатная система $p_{\phi}, \phi$ имеет особенность в точках $p_{\phi}= \pm \min \{\mu, \nu\}$. Она отличается от координатной системы на приведенном пространстве, использованной в [6]. Приведенная функция Гамильтона имеет вид

$$
\begin{aligned}
\widetilde{H}_{s}= & \frac{\left(1+r^{2}\right)^{2}}{8 m R^{2}} p_{r}^{2}+\frac{4 p_{\phi}^{2}}{a}+A_{s}\left(\mu^{2}+\nu^{2}-2 p_{\phi}^{2}+2 \sqrt{\mu^{2}-p_{\phi}^{2}} \sqrt{\nu^{2}-p_{\phi}^{2}} \cos \phi\right)+ \\
& +C_{s}\left(\mu^{2}+\nu^{2}-2 p_{\phi}^{2}-2 \sqrt{\mu^{2}-p_{\phi}^{2}} \sqrt{\nu^{2}-p_{\phi}^{2}} \cos \phi\right)+ \\
& +B_{s} \sqrt{\mu^{2}-p_{\phi}^{2}} \sqrt{\nu^{2}-p_{\phi}^{2}} \sin \phi+U(r) .
\end{aligned}
$$

В случае $\mu=0, \nu>0$ (или $\nu=0, \mu>0$ ) мы получаем для $O_{\beta_{0}}^{\prime}$ условия $u_{1}=u_{2}=$ $u_{3}=v_{1}=0$, поэтому $O_{\beta_{0}}^{\prime}=\mathbb{S}^{1}$ и $\widetilde{O}_{\beta_{0}}=$ pt. Приведенное фазовое пространство есть $T^{*} \mathbb{R}_{+}$с функцией Гамильтона

$$
\widetilde{H}_{s}=\frac{\left(1+r^{2}\right)^{2}}{8 m R^{2}}\left(p_{r}^{2}+\frac{\nu^{2}}{r^{2}}\right) .
$$

В случае $\mu=\nu=0$ мы получаем

$$
\widetilde{O}_{\beta_{0}}=O_{\beta_{0}}^{\prime}=\mathrm{pt}, \quad M=T^{*} \mathbb{R}_{+}, \quad \widetilde{H}_{s}=\frac{\left(1+r^{2}\right)^{2}}{8 m R^{2}} p_{r}^{2} .
$$


5.2. Задача двух частиц на пространстве $\mathbb{H}^{3}$. Алгебра Ли $\operatorname{so}(1,3)$ проста, поэтому мы не можем представить орбиты присоединенного действия групшы $\mathrm{SO}(1,3)$ как прямые произведения, подобно тому, как это сделано в п. 5.1. Однако динамические системы на сфере $\mathbb{S}^{3}$ и пространстве $\mathbb{H}^{3}$ связаны формальной заменой (см. п. 3.2 и [6]). Это мотивирует следующую конструкцию.

Пусть $L_{1}=X_{23}, L_{2}=X_{31}, L_{3}=X_{12}, Y_{1}, Y_{2}, Y_{3}$ - базис в алгебре $\operatorname{so}(1,3)$, соответствуюший полям Киллинга (4), а $L^{1}, L^{2}, L^{3}, Y^{1}, Y^{2}, Y^{3}$ - дуальный к нему базис. Пусть $\mathbf{p}=p_{1} L^{1}+p_{2} L^{2}+p_{3} L^{3}+p_{4} Y^{1}+p_{5} Y^{2}+p_{6} Y^{3}$ - произвольный элемент из so* $(1,3)$. Можно проверить прямым вычислением, что формулы

$$
I_{1}=p_{1}^{2}+p_{2}^{2}+p_{3}^{2}-p_{4}^{2}-p_{5}^{2}-p_{6}^{2}, \quad I_{2}=p_{1} p_{4}+p_{2} p_{5}+p_{3} p_{6}
$$

дают инварианты присоединенного действия группы $\mathrm{SO}(1,3)$. Так же, как в п. 5.1, мы получаем выражение функции Гамильтона через координаты на so* $(1,3)$ :

$$
H_{h}=\frac{\left(1-r^{2}\right)^{2}}{8 m R^{2}} p_{r}^{2}+\frac{1}{a} p_{4}^{2}+A_{h}\left(p_{2}^{2}+p_{3}^{2}\right)-C_{h}\left(p_{5}^{2}+p_{6}^{2}\right)+\frac{1}{2} B_{h}\left(p_{3} p_{5}-p_{2} p_{6}\right)+U(r) .
$$

Пусть $O_{\beta_{0}}$ - орбита коприсоединенного действия групшы $\mathrm{SO}(1,3)$, заданная уравнениями $I_{1}=\mu, I_{2}=\nu, \mu, \nu \in \mathbb{R}$. Стационарная подгруппа точки $\mathbf{x}_{0} \in F_{r}$ порождается элементом $L_{1}$. Действие этой подгруппы состоит в одновременном повороте в плоскостях $\left(p_{2}, p_{3}\right)$ и $\left(p_{5}, p_{6}\right)$. Подмногообразие $O_{\beta_{0}}^{\prime}$ задается уравнениями $I_{1}=\mu, I_{2}=\nu$, $p_{1}=0$. Следуюшие формулы задают координаты $p_{4}, \psi, \chi$ на нем:

$$
\begin{aligned}
& p_{2}=u \operatorname{ch} \psi \cos \chi+v \operatorname{sh} \psi \sin \chi, \quad p_{3}=v \operatorname{sh} \psi \cos \chi-u \operatorname{ch} \psi \sin \chi, \\
& p_{5}=v \operatorname{ch} \psi \cos \chi-u \operatorname{sh} \psi \sin \chi, \quad p_{6}=-u \operatorname{sh} \psi \cos \chi-v \operatorname{ch} \psi \sin \chi,
\end{aligned}
$$

где $p_{4}, \psi \in \mathbb{R}, \chi \in \mathbb{R}(\bmod 2 \pi)$, а $u$ и $v$ находятся из уравнений

$$
u^{2}-v^{2}=\mu+p_{4}^{2}, \quad u v=\nu
$$

Два решения уравнений (22) различаются знаком, поэтому нам достаточно выбрать одно из них. Действие стационарной групшы $\mathrm{SO}(2)$ соответствует врашению $\chi \rightarrow \chi+\xi$. Приведенное фазовое пространство $\widetilde{O}_{\beta_{0}}$ получается из $O_{\beta_{0}}^{\prime}$ “забыванием” координаты $\chi$. Пространство $\widetilde{O}_{\beta_{0}}$ диффеоморфно $\mathbb{R}^{2}$.

Для нахождения канонически-сопряженных координат на пространстве $\widetilde{O}_{\beta_{0}}$ мы используем вырожденные скобки Пуассона на so* $(1,3)$, которые соответствуют форме Кириллова. Эти скобки строятся для произвольной алгебры Ли $g$ следующим образом [20]

Пусть $\left\{e_{i}\right\}_{i=1}^{n}$ - базис алгебры $g,\left[e_{i}, e_{j}\right]=c_{i j}^{k} e_{k}$, a $\left\{x_{i}\right\}_{i=1}^{n}-$ координаты на $g^{*}$, соответствующие дуальному базису $\left\{e^{i}\right\}_{i=1}^{n}$. Пусть $f_{1}, f_{2}$ - произвольные гладкие функции на $g^{*}$. Тогда их скобки Пуассона имеют вид

$$
\left\{f_{1}, f_{2}\right\}=-\sum_{i, j, k=1}^{n} c_{i j}^{k} x_{k} \frac{\partial f_{1}}{\partial x_{i}} \frac{\partial f_{2}}{\partial x_{j}}
$$


Ограничение этих скобок на орбиту присоединенного действия невырожденно. Возврашаясь к нашему случаю и используя формулы

$$
\begin{gathered}
\psi=\frac{1}{4} \ln \left(\frac{\left(p_{2}-p_{6}\right)^{2}+\left(p_{5}+p_{3}\right)^{2}}{\left(p_{2}+p_{6}\right)^{2}+\left(p_{5}-p_{3}\right)^{2}}\right), \\
\chi=\frac{1}{2}\left(\operatorname{arctg}\left(\frac{p_{5}-p_{3}}{p_{2}+p_{6}}\right)-\operatorname{arctg}\left(\frac{p_{5}+p_{3}}{p_{2}-p_{6}}\right)\right), \\
{\left[L_{i}, L_{j}\right]=\sum_{k=1}^{3} \varepsilon_{i j k} L_{k}, \quad\left[Y_{i}, Y_{j}\right]=-\sum_{k=1}^{3} \varepsilon_{i j k} L_{k}, \quad\left[L_{i}, Y_{j}\right]=\sum_{k=1}^{3} \varepsilon_{i j k} Y_{k},}
\end{gathered}
$$

прямыми вычислениями мы получаем следующие соотношения:

$$
\left\{p_{4}, \psi\right\}=1, \quad\left\{p_{4}, \chi\right\}=0, \quad\{\psi, \chi\}=0 .
$$

Тогда симплектическая структура на $\widetilde{O}_{\beta_{0}}$ задается формулой $d p_{4} \wedge d \psi$. Из (21) мы получаем

$$
\begin{aligned}
p_{2}^{2}+p_{3}^{2} & =\frac{1}{2}\left(\mu+p_{4}^{2}+\sqrt{\left(\mu+p_{4}^{2}\right)^{2}+4 \nu^{2}} \operatorname{ch} 2 \psi\right), \\
p_{5}^{2}+p_{6}^{2} & =\frac{1}{2}\left(-\mu-p_{4}^{2}+\sqrt{\left(\mu+p_{4}^{2}\right)^{2}+4 \nu^{2}} \operatorname{ch} 2 \psi\right), \\
p_{3} p_{5}-p_{2} p_{6} & =\frac{1}{2} \sqrt{\left(\mu+p_{4}^{2}\right)^{2}+4 \nu^{2}} \operatorname{sh} 2 \psi .
\end{aligned}
$$

Вводя новые канонически-сопряженные координаты $p_{\phi}=p_{4} / 2, \phi=2 \psi$, мы получаем из (20) окончательную формулу приведенной функции Гамильтона

$$
\begin{aligned}
\widetilde{H}_{h}= & \frac{\left(1-r^{2}\right)^{2}}{8 m R^{2}} p_{r}^{2}+\frac{4 p_{\phi}^{2}}{a}+A_{h}\left(\frac{\mu}{2}+2 p_{\phi}^{2}+2 \sqrt{\left(\frac{\mu}{4}+p_{\phi}^{2}\right)^{2}+\frac{\nu^{2}}{4}} \operatorname{ch} \phi\right)+ \\
& +C_{h}\left(\frac{\mu}{2}+2 p_{\phi}^{2}-2 \sqrt{\left(\frac{\mu}{4}+p_{\phi}^{2}\right)^{2}+\frac{\nu^{2}}{4}} \operatorname{ch} \phi\right)+ \\
& +B_{h} \sqrt{\left(\frac{\mu}{4}+p_{\phi}^{2}\right)^{2}+\frac{\nu^{2}}{4}} \operatorname{sh} \phi+U(r) .
\end{aligned}
$$

\section{6. ЗАКЛЮЧЕНИЕ}

Мы построили представление квантово-механического гамильтониана системы двух частиц на пространствах $\mathbb{S}^{n}$ и $\mathbb{H}^{n}$, явно учитываюшее имеюшиеся симметрии. На основе полученного выражения в следуюшей работе будет установлена квазиточнорешаемость соответствуюшей спектральной задачи для ряда потенциалов. Явный вид приведенной функции Гамильтона в канонических координатах, полученный в [6] аналитическими вычислениями на ЭВМ, был использован там для анализа отсутствия столкновений частиц. В настоящей работе мы прояснили, в частности, происхождение явного вида приведенной функции Гамильтона и ее связь с квантово-механическим гамильтонианом. 


\section{Список литературы}

[1] Дж. Вольф. Пространства постоянной кривизны. М.: Наука, 1982.

[2] J. Moser. Commun. Pure Appl. Math. 1970. V. 23. P. 609.

[3] Ю. C. Ocunoв. УМH. 1972. Т. 27. № 2. С. 101.

[4] Y.S. Osipov. Celest. Mech. 1977. V. 16. P. 191.

[5] E. A. Belbruno. Celest. Mech. 1977. V. 15. P. 467.

[6] A. V. Shchepetilov. J. Phys. A. 1998. V. 31. P. 6279; 1999. V. 32. P. 1531.

[7] M. Ikeda, N. Katayama. Tensor. 1982. V. 38. P. 37.

[8] N. Katayama. Nuovo Cimento B. 1990. V. 105. P. 113; 1992. V. 107. P. 763; 1993. V. 108. P. 657.

[9] Я. И. Грановский, А.С. ЖКеданов, И. М. Луценко. ТМФ. 1992. Т. 91. С. 207; С. 396.

[10] V.S. Otchik. On the two Coulomb centres problem in a spherical geometry. In: Proc. Intern. Workshop on Symmetry Methods in Physics. V. 2. Eds. A. N. Sissakian, G. S. Pogosyan, S. I. Vinitsky. Dubna: JINR, 1994. P. 384.

[11] V. A. Chernoivan, I. S. Mamaev. Regular and chaotic dynamics. 1999. V. 4. № 2. P. 112.

[12] A. В. Борисов, И. С. Мамаев. Пуассоновы структуры и алгебры Ли в гамильтоновой механике. Ижевск: Редакция журнала "Регулярная и хаотическая динамика", 1999.

[13] В. И. Арнольд. Математические методы классической механики. М.: Наука, 1989.

[14] А. В. Щепетилов. ТМФ. 1999. Т. 118. С. 248.

[15] М. Рид, Б. Саймон. Методы современной математической физики. Т. 1. Функциональный анализ. М.: Мир, 1977.

[16] И. М. Олейник. Мат. заметки. 1994. Т. 55. С. 218.

[17] А. В. Щепетилов. ТМФ. 1996. Т. 109. С. 395.

[18] C. Хелгасон. Группы и геометрический анализ. М.: Мир, 1984.

[19] A. А. Кириллов. Элементы теории представлений. М.: Наука, 1972.

[20] В. В. Трофимов, А. Т. Фоменко. Алгебра и геометрия интегрируемых гамильтоновых дифференциальных уравнений. М.: Факториал, 1995.

Поступила в редакцию $12 . X I .1999$ г., после доработки 3.IV.2000 г. 